\title{
Gleason score predicted mortality rate to 20 years for untreated early prostate cancer
}

Albertsen PC, Hanley JA, Fine J. 20-year outcomes following conservative management of clinically localized prostate cancer. JAMA 2005;293:2095-101.

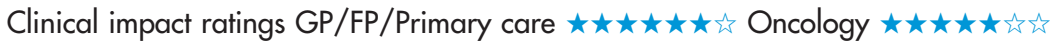

\section{In patients with localised prostate cancer, what is the 20 year survival rate?}

\section{METHODS}

L-

Design: inception cohort followed for a median of 24 years (range 16-33 y).

Setting: 35 acute care hospitals and 2 Veterans Affairs medical centres in Connecticut, USA.

Patients: 767 men 55-74 years of age (mean age $69 \mathrm{y}$ ) diagnosed with clinically localised prostate cancer between 1

I- January 1971 and 31 December 1984 who were treated with observation or immediate or delayed androgen withdrawal therapy. Exclusion criteria were surgery, radiation therapy, brachytherapy, known metastatic disease, concomitant cancer, or survival $<6$ months.

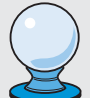

Prognostic factors: age at diagnosis, treatment method, comorbid conditions, and tumour grade according to Gleason score (range 2-10; $10=$ most undifferentiated).

Outcomes: death from prostate cancer or other competing causes.

\section{MAIN RESULTS}

Prostate cancer mortality rates were 33 deaths per 1000 person years ( $95 \%$ CI 28 to 38 ) in the first 15 years of follow up and 18 deaths per 1000 persons years (CI 10 to 29) after 15 years. After adjusting for Gleason score, the rates before and after 15 years did not differ (rate ratio 1.1, CI 0.6 to 1.9). Men with low grade tumours had low risk of dying from prostate cancer over the next 20 years; the mortality rate increased with increasing Gleason score (table).

For correspondence: $\mathrm{Dr} \mathrm{P} \mathrm{C}$ Albertsen, University of Connecticut Health Center, Farmington, CT, USA. albertsen@nso.uchc.edu

Source of funding: Agency for Healthcare Research and Quality

Death from prostate cancer by fumour grade at diagnosis*

\begin{tabular}{lll}
\hline Gleason score & $\begin{array}{l}\text { Number of } \\
\text { prostate cancer } \\
\text { deaths }\end{array}$ & $\begin{array}{l}\text { Deaths per } 1000 \text { person years(95\% } \\
\text { Cl) }\end{array}$ \\
\hline $2-4$ & 10.7 & $6(2$ to 11$)$ \\
5 & 16.6 & $12(6$ to 19$)$ \\
6 & 83.4 & $30(23$ to 37$)$ \\
7 & 63.4 & $65(49$ to 83$)$ \\
$8-10$ & 54.4 & $121(90$ to 156$)$ \\
\hline
\end{tabular}

*Gleason score range $2-10 ; 10=$ most undifferentiated.

\section{CONCLUSIONS}

In patients with localised prostate cancer, mortality rates were similar before and after 15 years when tumour grade at diagnosis was taken into account. Gleason score predicted a 20 fold difference in survival.

\section{Commentory}

The prostate cancer mortality rate did not increase after 15 years in the study by Albertsen et al, unlike another large, recently reported cohort study of men with prostate cancer who were managed conservatively. 'Because few patients in either cohort lived $>15$ years, this difference is less important than the substantial difference in prostate cancer mortality over the first 15 years $(33 v 15$ per 1000 person- $y$ in the Albertsen and Johansson studies, respectively). This difference is probably due to the higher proportion of men with poorly differentiated tumours in the study by Albertsen et al.

This hypothesis is supported by the prostate cancer mortality rate of 16 per 1000 person years in the conservatively managed group of the SPCG-4 trial comparing radical prostatectomy with watchful waiting, which excluded men with high grade tumours. ${ }^{2}$ The outcomes of watchful waiting in these 3 studies represent "worst case scenarios" for contemporary men with prostate cancers of similar grades diagnosed because of elevated concentrations of serum prostate specific antigen (PSA). The effects of lead time and overdiagnosis attributable to PSA screening will improve the apparent prognosis regardless of treatment. However, it is hard to estimate the effect of screening on the number needed to treat (NNT) observed in the trial, where about 19 operations were required to prevent a prostate cancer death at 10 years.

Although lead time and overdiagnosis would increase the NNT by improving the results of watchful waiting, perhaps screening would decrease the NNT by improving the results of surgery if earlier detection and treatment prevented additional deaths. Most importantly, we do not know the effect of early detection on the subset of poorly differentiated cancers that contribute most to mortality. The PIVOT trial of radical prostatectomy versus watchful waiting enrolled men largely discovered by screening, including those with poorly differentiated cancers, and should help fill this knowledge gap by the end of the decade. ${ }^{3}$

Michael J Barry, MD Massachusetts General Hospital Boston, Massachusetts, USA

1 Johansson JE, Andrén $\mathrm{O}$, Andersson SO, et al. Natural history of early, localized prostate cancer. JAMA 2004;291:2713-9.

2 Bill-Axelson A, Holmberg L, Ruutu M, et al. Radical prostatectomy versus watchful waiting in early prostate cancer. N Engl J Med 2005;352:197784.

3 Wilt TJ, Brawer MK. The Prostate Cancer Intervention Versus Observation Trial: a randomized trial comparing radical prostatectomy versus expectant management for the treatment of clinically localized prostate cancer. J Urol $1994 ; 152: 1910-4$ 